\title{
Etude de l'effet rémanent de Mavi MNPV dans un champ de niébé
}

\author{
A. KINDOZANDJI ${ }^{1 *}$, B. D. DATINON ${ }^{1}$, K. AMEVOIN ${ }^{2}$, A. N. NONDICHAO ${ }^{1}$, \\ A. I. GLITHO ${ }^{2}$ et M. TAMÓ ${ }^{1}$ \\ ${ }^{1}$ International Institute of Tropical Agriculture 08 BP 0932 Tri-postal Cadjehoun, Cotonou, Bénin. \\ ${ }^{2}$ Université de Lomé, Faculté des Sciences, BP 1515, Lomé, Togo. \\ ${ }^{*}$ Corresponding author; E-mail: kinauguste@yahoo.fr
}

\section{RESUME}

Le niébé est l'une des légumineuses alimentaires la plus importante dans toutes les régions de l'Afrique Sub-saharienne. L'objectif de ce travail était d'étudier l'effet rémanent de Mavi Multinucléo polyhedrovirus (Mavi MNPV) après la récolte du niébé au champ. Le champ a été traité 5 fois au biopesticide Mavi MNPV à la fréquence de sept jours. Deux semaines après la récolte, deux plants ainsi que les graines de chaque traitement sur chaque unité parcellaire ayant reçu les traitements de Mavi MNPV de $10^{7}, 10^{8}$ et $10^{9}$ virions $/ \mathrm{ml}$ ont été collectés et amenés au laboratoire avec le substrat de développement de la plante (sable). Aucune des formulations issues des substrats sable, racines, feuilles, tiges et graines n'a affecté la mortalité des larves L2 et L3 de Maruca vitrata et aucune différence significative n'a été observée entre les traitements et le témoin. Quels que soient les traitements, le taux d'émergence des adultes est élevé ; ce qui signifie que la variation de la concentration de Mavi MNPV ne conditionne pas sa présence dans le sol, les tiges, les feuilles, les racines et les graines. Le biopesticide Mavi MNPV n'est pas persistant dans la nature.

(C) 2020 International Formulae Group. All rights reserved.

Mots clés : Niébé, Mavi MNPV, Maruca vitrata, Effet rémanent

\section{Study of the residual effect of Mavi MNPV in a cowpea field}

\begin{abstract}
Cowpea is one of the most important food legumes in all regions of Sub-Saharan Africa. The objective of this research work was to study the persistence effect of Mavi Multinucleus polyhedrovirus (Mavi MNPV) after field harvest of the cowpea. The field was treated 5 times with Mavi MNPV biopesticide at seven days. Two weeks after harvest, two plants as well as the seeds from each treatment on each plot unit that received Mavi MNPV treatments of $10^{7}, 10^{8}$ and $10^{9}$ virions / $\mathrm{ml}$ were removed and brought to the laboratory with the plant development substrate (sand). None of the formulations from sand, root, leaf, stem and seed substrates did not affect the mortality of L2 and L3 larvae of Maruca vitrata and no significant difference was observed between treatments and control. Regardless of the treatments, the rate of emergence of adults is high; this means that the variation in the concentration of Mavi MNPV does not condition its presence in soil, stems, leaves, roots and seeds. Mavi MNPV biopesticide is not persistent in nature.
\end{abstract}

(C) 2020 International Formulae Group. All rights reserved.

Keywords: Cowpea, Mavi MNPV, Maruca vitrata, Residual effect. 


\section{INTRODUCTION}

Le niébé, Vigna unguiculata (L.) Walp. est l'une des principales légumineuses alimentaires mondiales. Importante source de protéines, d'hydrates de carbone et de nombreux autres éléments nutritifs essentiels (El-Jasser, 2011), il joue un rôle majeur dans l'alimentation humaine dans les savanes arides de l'Afrique de l'Ouest où peu de gens ont accès aux protéines animales (Phillips et al., 2003). Le niébé occupe la première place du point de vue nutritionnel, agronomique, économique et socio-culturel. Ses multiples centres d'intérêts que présentent la culture et sa consommation font de sa production une garantie à la sécurité alimentaire et une contribution à la réduction de la pauvreté aussi bien au Bénin que dans tous les pays sousdéveloppés (Atachi et al., 1984). Toutes les parties de la plante sont consommées, car elles sont riches en nutriments et en fibres. Le niébé n'est pas seulement source de protéines végétales, il est aussi source de revenus financiers pour l'humain; c'est une culture d'importance économique, sociale et alimentaire. Cependant, sa production est confrontée à plusieurs contraintes qui limitent son rendement. Il s'agit des aléas climatiques, les mauvaises pratiques culturales, du faible potentiel de production de certaines variétés cultivées, les adventices, les maladies et surtout des insectes nuisibles. En effet, les pertes occasionnées par les différentes contraintes biologiques (insectes ravageurs, nématodes, maladies et adventices) sont évaluées à près de 300 millions de dollars américains par an (NRI, 2012). Au nombre des ravageurs redoutables du niébé, figure la foreuse des fleurs et des gousses, Maruca vitrata Fabricius (Lepidoptera: Crambidae) largement distribuée dans les régions tropicales et subtropicales de l'Asie, de l'Amérique latine et de l'Afrique (Liao et Lin, 2000). Elle cause des dégâts sévères allant jusqu'à la perte quasi totale des récoltes (Seri-kouassi et al., 2004). Ainsi, pour faire face à cela, les producteurs ont recours aux pesticides chimiques de synthèse. L'application abusive de ces derniers sur cette culture ainsi que les mauvaises conditions de stockage et de conservation, menacent la santé des producteurs directement exposés, et même celle des consommateurs des produits non conformes aux limites maximales de résidus (LMRs) du Codex Alimentarius (FAO, 2002). Les études menées sur la dispersion des pesticides (aldrine, toxaphène, DDT, chlordane, dieldrine, endrine, heptachlore et mirex) dans l'environnement ont prouvé la présence de ces produits toxiques pour l'humain dans plusieurs points de la biosphère tels que les sols, les eaux, les chaînes trophiques (Ramezani et al., 2008). Les pesticides renferment des substances dangereuses et toxiques même à très faibles doses (Carlos, 2006). Selon Gomgnimbou et al. (2009), leur utilisation est source des risques d'ordre sanitaire, de pollution des eaux et des sols et de développement de résistance chez les parasites ciblés (Ouédraogo, 2004 ; Bass et al.,2015 ; Narayan et al., 2017), Les effets des pesticides sur l'environnement et la santé ne sont donc pas compatibles avec une exploitation durable des agro-écosystèmes comme mentionné par plusieurs auteurs (Deguine et Ferron, 2004 ; Narayan et al., 2017). Les producteurs n'attendent même pas le délai de carence des produits chimiques avant de faire leur récolte. Toutefois, un composé qui se dégraderait en quelques jours à l'extérieur peut demeurer actif pendant des années (Hénault-heltier, 2015). Selon le même auteur, il faut en moyenne entre 30 et 100 jours à la plupart des pyréthrinoïdes pour se décomposer dans un sol en présence d'oxygène. À l'intérieur d'un élévateur à grain ou d'un tunnel de métro, ce délai peut se prolonger jusqu'à un an, et certaines formulations dotées d'effets résiduels (comme celles servant à protéger le bois des termites) peuvent persister jusqu'à cinq ans. La recherche a montré qu'un mélange d'organophosphorés et de pyréthrinoïdes peut faire augmenter de 140 à 170 fois la toxicité chez le poisson en raison des impacts sur le système de détoxication des animaux exposés à de multiples composés (Hénault-heltier, 2015). Selon le même auteur, leur combinaison réduit le nombre de spermatozoïdes chez l'homme. En effet, Plusieurs alternatives aux produits chimiques de synthèse existent. Il faut envisager d'autres solutions moins toxiques comme les interventions physiques, les actions 
biologiques, les pesticides à faible impact ou les extraits complexes de plantes naturelles afin de contrer la résistance croissante des insectes aux pesticides chimiques de synthèse. La lutte biologique implique l'usage d'auxiliaires dont les prédateurs, les parasitoïdes et des microorganismes (Van Lenteren et al., 2006). Les biopesticides testés jusqu'à présent sont d'origine végétale, animale ou microbiologique. La virulence du baculovirus Multi Nucleo polyhedrovirus (Mavi MNPV) a été prouvée. Des travaux réalisés sur l'efficacité de Mavi MNPV comme biopesticide au laboratoire et au champ au Bénin, au Burkina Faso, au Niger et au Nigeria se sont révélés prometteurs (Tamò et al., 2012). De même l'efficacité de ce produit a été prouvé au champ (Sokame et al., 2015 ; Toffa Mehinto 2014). C'est ainsi que les champs traités aux différentes concentrations de Mavi Multinucléo polyhedrovirus (Mavi MNPV) à la station de l'Institut International d'Agriculture Tropical du Bénin ne faisaient pas toujours objet d'étude pour apprécier les résidus de pesticides après récolte. Il a été constaté aussi que très peu d'études ont été menées pour évaluer l'effet rémanent du baculovirus Mavi MNPV après son utilisation dans les champs de niébé. C'est pourquoi deux semaines après la récolte, les prélèvements des substrats sols, feuilles, tiges, racines et graines ont focalisé l'attention d'une recherche sur les résidus de ce produit. L'objectif de l'étude était d'étudier les effets rémanents du baculovirus Mavi MNPV après récolte du niébé au champ.

\section{MATERIEL ET METHODES Milieux d'étude}

Les travaux de laboratoire ont été conduits à la station de l'Institut International d'Agriculture Tropicale (IITA-Bénin) dans le laboratoire d'entomologie de l'IITA-Bénin de mai à décembre 2019 sous la température moyenne de $26,75 \pm 0,27{ }^{\circ} \mathrm{C}$ et l'humidité relative moyenne était de $54,45 \pm 1,99 \%$.

L'IITA-Bénin est située à Togoudo $\left(06^{\circ} 2155^{\prime} \mathrm{N}, 02^{\circ} 2550^{\prime} \mathrm{E}\right)$ dans la commune d'Abomey-Calavi, département de l'Atlantique, au Sud du Bénin. Il est situé à 12 $\mathrm{km}$ au Nord-Ouest de Cotonou (capitale économique de la République du Bénin) et à
2,5 km environ de la voie inter État CotonouBohicon avec une altitude de $15 \mathrm{~m}$ au-dessus de la mer.

\section{Dispositif expérimental}

Le dispositif expérimental au champ était un bloc aléatoire complet. Chaque unité parcellaire mesurait $7 \mathrm{~m} \times 5 \mathrm{~m}$ soit une superficie de $35 \mathrm{~m}^{2}$. La variété utilisée a un cycle de 65 à 70 jours. Le dernier traitement a été effectué le $58^{\text {ème }}$ après semis. La récolte a été réalisée 14 jours après le dernier traitement. L'échantillonnage des substrats sable, feuilles, tiges racines et graines a été réalisé 14 jours après la récolte. Cette période a été choisie en tenant compte des travaux realisés par Ben et al. (2005). L'essai a été conduit au laboratoire suivant un dispositif de bloc complètement aléatoire, incluant les facteurs suivants: des stades de développement de l'insecte $\mathrm{L}_{2}$ et $\mathrm{L}_{3}$ à tester, les substrats de sable, graines, racines, feuilles, tiges prélevés dans un champ traité avec Mavi MNPV, $106 \mathrm{ml}$ à $10^{7}, 10^{8}$ et $10^{9}$ virions $/ \mathrm{ml}$.

\section{$\checkmark \quad$ Test avec le substrat sable}

Le sable prélevé au niveau des plants traités à Mavi MNPV $106 \mathrm{ml}$ à $10^{7}, 10^{8}$ et $10^{9}$ virions $/ \mathrm{ml}$ par hectare dans 115 litres d'eau ont été choisis par bloc pour faire l'expérience. Le substrat collecté sur les parcelles a été regroupé par traitement et pésé. Ainsi, $300 \mathrm{~g}$ de ce substrat ont été introduits dans des boîtes auxquelles 1 litre d'eau a été ajouté. Le mélange a été agité manuellement, macéré pendant 24 heures, puis filtré. La solution de substrat obtenue a été utilisée pour faire germer le niébé sain. Deux de ces graines prégermées ont été déposées dans des boîtes de $30 \mathrm{~cm}^{3}$ contenant soit une larve $\mathrm{L}_{2}$ ou $\mathrm{L}_{3}$ de $M$. vitrata. Chaque lot de 30 larves traitées a été répété quatre fois.

\section{$\checkmark \quad$ Test avec le substrat graines}

Témoin absolu sans aucun traitement: Les graines proviennent d'un champ sans traitement de biopesticide et de produits chimiques.

Tests avec Mavi MNPV: les graines issues des parcelles traitées avec Mavi MNPV $106 \mathrm{ml}$ à $10^{7}, 10^{8}$ et $10^{9}$ virions $/ \mathrm{ml}$ ont servi de substrat pour l'expérimentation. Ces graines étaient trempées dans l'eau pendant 4 heures. Ensuite, elles ont été retirées et séchées par ventilation 
dans les conditions ambiantes pendant 24 heures. Deux de ces graines prégermées ont été déposées dans des boîtes de $30 \mathrm{~cm}^{3}$ contenant soit une larve $\mathrm{L}_{2}$ ou $\mathrm{L}_{3}$ de $M$. vitrata. Chaque lot de 30 larves traitées a été répété quatre fois.

$\checkmark$ Test avec le mélange racines, feuilles et tiges de niébé

Les extraits totaux par concentration de Mavi MNPV ont été obtenus par macération des produits de la mouture des feuilles, tiges et racines après séchage. Ainsi, $300 \mathrm{~g}$ de ce substrat ont été introduits dans des boîtes auxquelles il a été ajouté 1 litre d'eau. Le mélange a été soumis à une agitation manuelle, puis macéré pendant 24 heures, puis filtré. Le filtra obtenu a été utilisée pour faire germer le niébé sain. Deux de ces graines prégermées ont été déposées dans des boîtes de $30 \mathrm{~cm}^{3}$ contenant soit une larve $\mathrm{L}_{2}$ ou $\mathrm{L}_{3}$ de $M$. vitrata. Chaque lot de 30 larves traitées a été répété quatre fois.

Suivi des expériences et paramètres mesurés: un suivi a été effectué tous les jours durant tout l'essai afin de relever les mortalités larvaires et l'émergence des adultes de $M$. vitrata.

\section{Analyse des données}

L'analyse descriptive des données collectées a été faite à l'aide du tableau Excel. Les comparaisons statistiques entre les différents traitements ont été réalisées à l'aide d'une analyse de variance (ANOVA) par la procédure <<General Linear Models〉>(GLM) avec le logiciel SAS 9.2. Lorsque l'analyse de variance révèle des différences significatives, le test de séparation des moyennes de StudentNewman-Keuls (SNK) a été appliqué au seuil de $5 \%$. Les graphes ont été réalisés avec le logiciel JMP version Pro 14 SAS Intituté Inc., Cary, NC, 1989-2019.

\section{RESULTATS}

Effets du substrat sable de parcelle de chaque traitement sur la mortalité des larves $\mathrm{L}_{2}$ et $\mathrm{L}_{3}$ de $M$. vitrata

Pour le substrat Sable, le taux de mortalité de $\mathrm{L}_{2}$ a varié de $13,33 \% \pm 5,77$ (Témoin) à 17,77 $\pm 1,11 \%$ (Mavi MNPV $10^{7}$ virions $/ \mathrm{ml}$ ). L'analyse de variance a révélé qu'il n'existe pas de différence significative entre les traitements de Mavi MNPV et le témoin $(P=0,6402)$ (Figure 1). Quant à $\mathrm{L}_{3}$, le taux de mortalité a varié de $11,11 \pm 4,0 \%$ (Témoin) à $15,55 \pm 1,11 \%$ (Mavi MNPV $10^{9}$ virions/ml pour tous les traiements y compris le témoin. L'analyse de variance n'a révélé aucune différence significative entre les traitements de Mavi MNPV et le témoin $(\mathrm{P}=$ 0,6325) (Figure 1).

Effets du substrat sable de parcelle de chaque traitement sur l'émergence des adultes des larves $\mathrm{L}_{2}$ et $\mathrm{L}_{3}$ de $M$. vivrata

Le taux moyen d'émergence des adultes issu des larves $\mathrm{L}_{2}$ de l'insecte test pour le traitement témoin était de 78,88 $\pm 6,18 \%$. Celui des traitements a varié entre $70 \pm 3,33 \%$ à $75,55 \pm 7,77 \%$. Aucune différence significative n'a été constatée entre le témoin et les traitements. Quant à $\mathrm{L}_{3}$, le taux moyen d'émergence des adultes de l'insecte test pour le traitement témoin était de $62,22 \pm 4,84 \%$. Celui des traitements a varié entre $56,66 \pm 1,92 \%$ à $65,55 \pm 6,75 \%$. Aucune différence significative n'a été constatée entre le témoin et les traitements $(P=0,1487)$ (Figure 2).

Effets des graines issues des parcelles traitées au Mavi MNPV sur la mortalité des larves $L_{2}$ et $L_{3}$

Le taux moyen de mortalité pour le témoin $\mathrm{L}_{2}$ était de $16,66 \pm 6,93 \%$. Il a varié entre 14,44 $\pm 2,93 \%$ (Mavi MNPV $10^{8}$ virions $/ \mathrm{ml}$ ) à 22,22 $\pm 2,93 \%$ (Mavi MNPV $10^{7}$ virions $/ \mathrm{ml}$ ). Aucune différence significative n'a été observée pour les traitements Mavi MNPV pour les larves $\mathrm{L}_{2}$ et le témoin $(P=0,5637)$ (Figure 5). En ce qui concerne L3, le taux moyen de mortalité pour le témoin était de $8,89 \pm 2,22 \%$. Celui des traitements a varié de $12,22 \pm 1,11 \%$ à $14,44 \pm 2,22 \%$. L'analyse de variance n'a révélé aucune différence significative entre les traitements de Mavi MNPV et le témoin $(P=0,2089)$ (Figure 3 ).

Effets des graines issues des parcelles traitées au Mavi MNPV sur l'émergence des adultes des larves $L_{2}$ et $L_{3}$ de $M$. vitrata

Le taux d'émergence des adultes issu de $\mathrm{L}_{2}$ de l'insecte test pour le traitement témoin 
était de $78,88 \pm 6,18 \%$. Celui des traitements a varié de $74,44 \pm 1,11 \%$ à $75,55 \pm 1,11 \%$. L'analyse de variance a révélé que le taux d'émergence des adultes n'a pas connu de différence significative ( $P=0,0022)$ (Figure 4). S'agissant des adultes issus de $\mathrm{L}_{3}$, le taux d'émergence des adultes pour le témoin était de $80,00 \pm 3,33 \%$. Celui des traitements a varié de $75,55 \pm 2,93 \%$ à $78,88 \pm 2,22 \%$. L'analyse de variance n'a révélé aucune différence significative selon le test SNK $(P=0,3617)$, (Figure 4).

Effets de substrat mélange de racines, feuilles et tiges de niébé traité sur la mortalité des larves $L_{2}$ et $L_{3}$ de $M$. vitrata

En ce qui concerne le substrat mélange de racines, tiges et feuilles, le taux moyen de mortalité du témoin était de 14,44 \pm 4,84\% pour les larves $\mathrm{L}_{2}$ et celui des traitements a varié de $16,66 \pm 3,33 \%$ à $21,11 \pm 4,00 \%$. Cependant, l'analyse de variance n'a noté aucune différence significative selon le test SNK pour les traitements Mavi MNPV comparés au témoin $(P=0,6920)$ (Figure 5).
S'agissant des larves $\mathrm{L}_{3}$, le taux moyen de mortalité du témoin était de $12,22 \pm 1,11 \%$ et celui des traitements a varié de 14,44 $\pm 2,93$ à $16,66 \pm 3,33 \%$. L'analyse de variance n'a noté aucune différence significative selon le test SNK pour les traitements Mavi MNPV comparés au témoin $(P=0,3888)$ (Figure 5).

Effets de substrat mélange de racines, feuilles et tiges de niébé traité sur l'émergence des adultes des larves $L_{2}$ et $L_{3}$ de $M$. vitrata

Le taux d'émergence des adultes issus $\mathrm{L}_{2}$ pour le témoin était de 74,44 22,22\%. Quant aux traitements, il a varié de $67,77 \pm 4,00 \%$ à 73,33 $\pm 3,84 \%$. L'analyse de variance a montré qu'il n'existe pas de différence significative $(P$ $=0,1148$ ) entre le taux d'émergence pour le traitement de Mavi MNPV et le témoin (Figure 6). Quant à L3, Le Taux d'émergence des adultes pour le témoin était de $74,44 \pm 2,22 \%$. Celui des traitements a varié de $64,44 \pm 4,00 \%$ à 72,22 \pm $4,00 \%$. L'analyse de variance n'a révélé aucune différence significative $(\mathrm{P}=0,3086)$, (Figure 6).

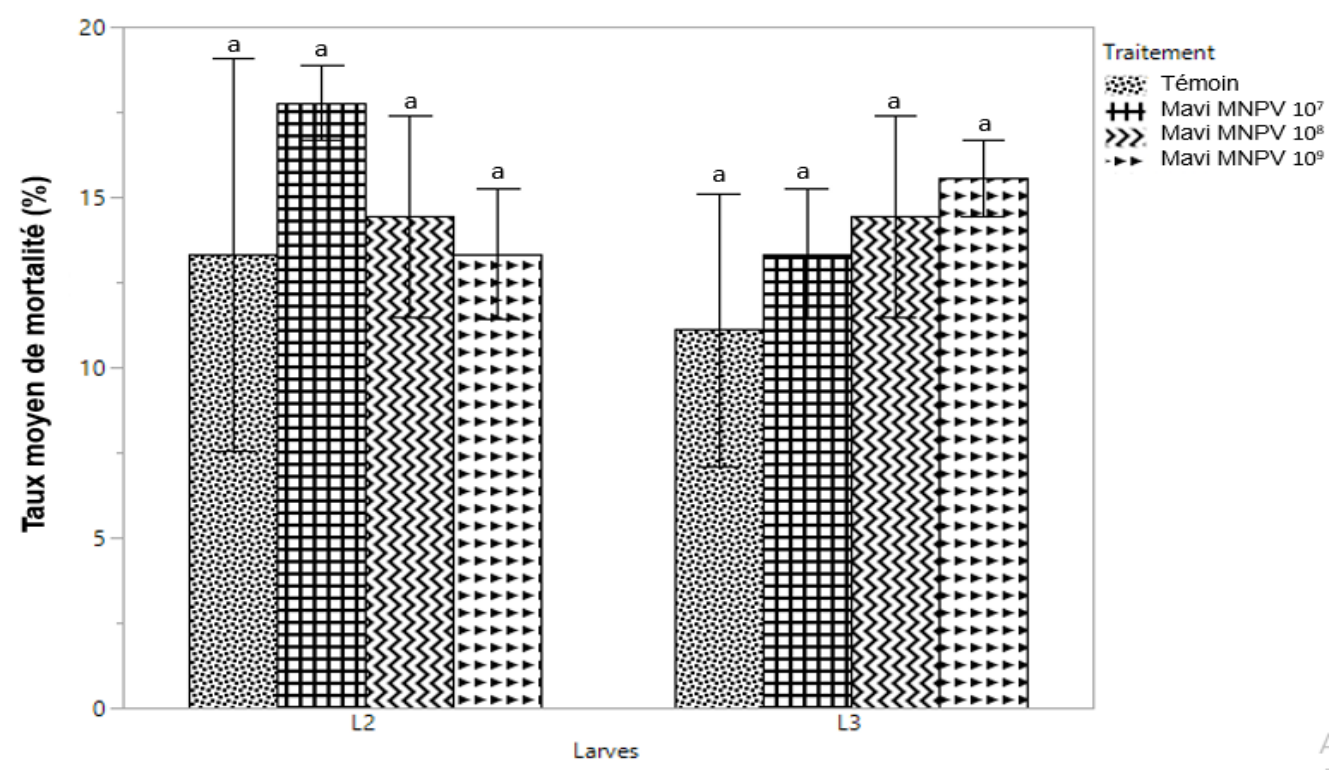

Figure 1 : Taux moyens de mortalité des larves $\mathrm{L}_{2}$ et $\mathrm{L}_{3}$ sur le substrat sable issu des parcelles traitées. 


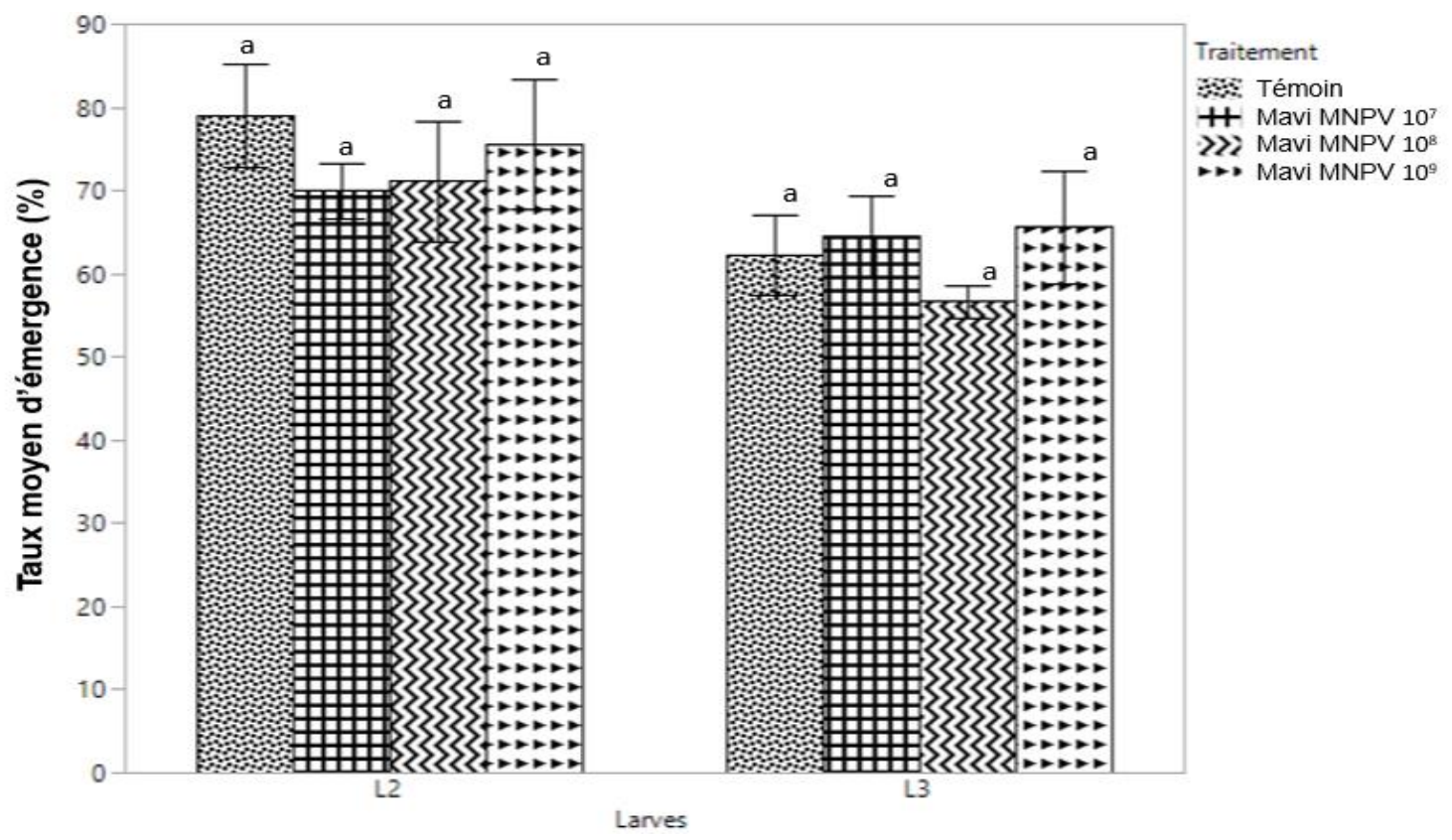

Figure 2 : Taux moyens d'émergence des adultes issus des larves $\mathrm{L}_{2}$ et $\mathrm{L}_{3}$ sur le substrat sable.

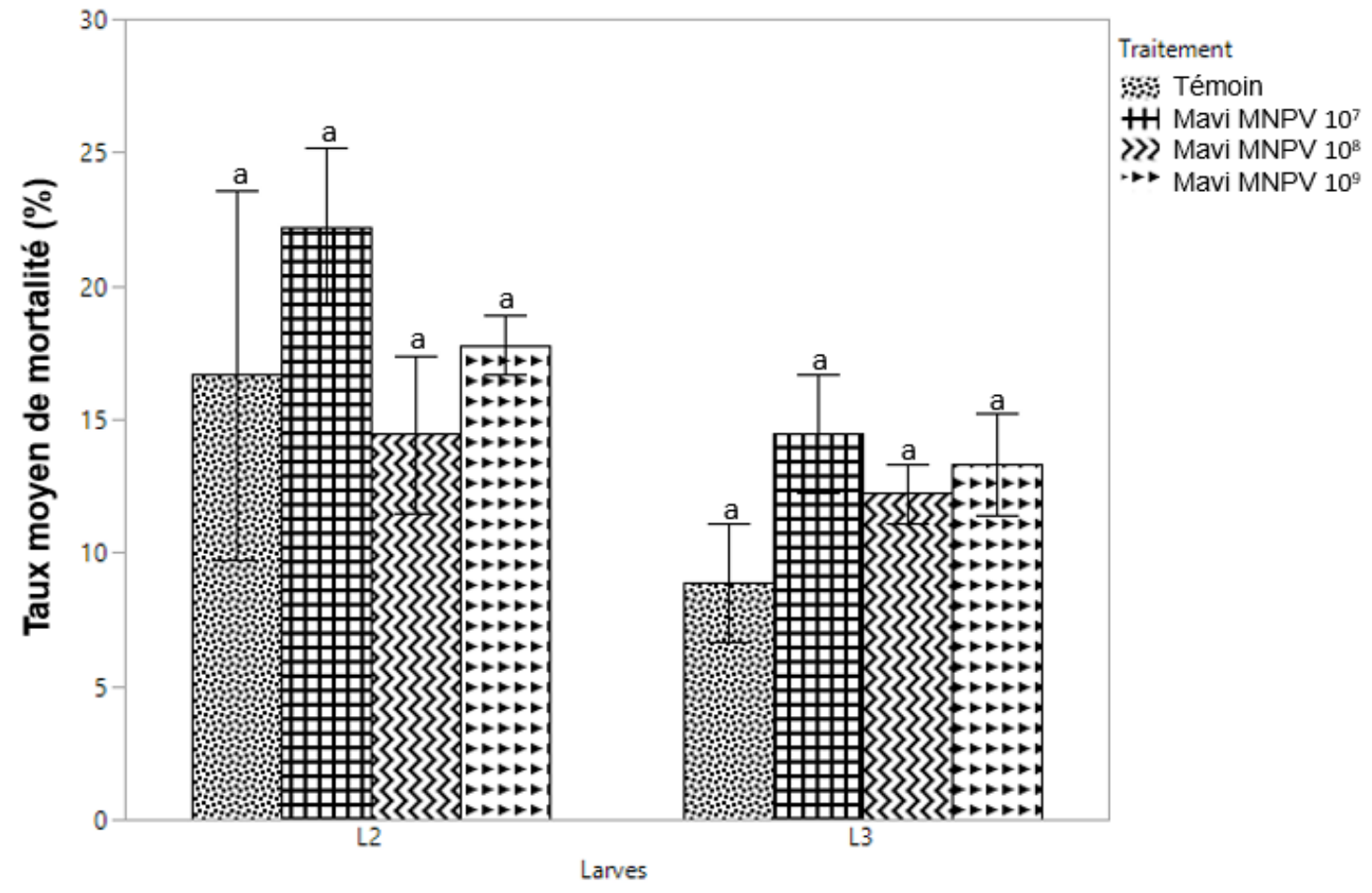

Figure 3 : Taux moyens de mortalité des larves $L_{2}$ et $L_{3}$ sur le substrat Graines. 


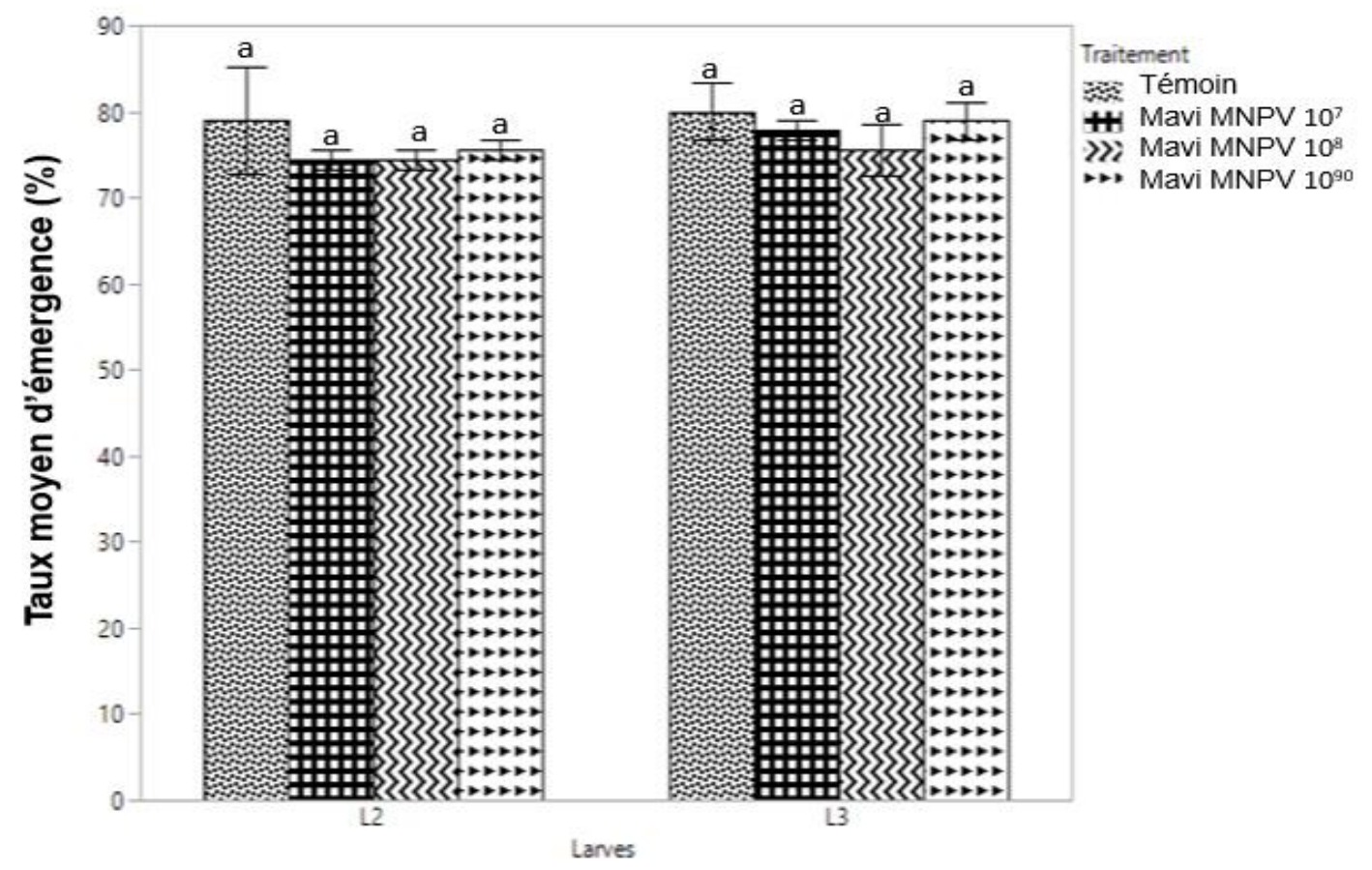

Figure 4 : Taux moyens d'émergence des adultes issus des larves $\mathrm{L}_{2}$ et $\mathrm{L}_{3}$ sur le substrat Graines.

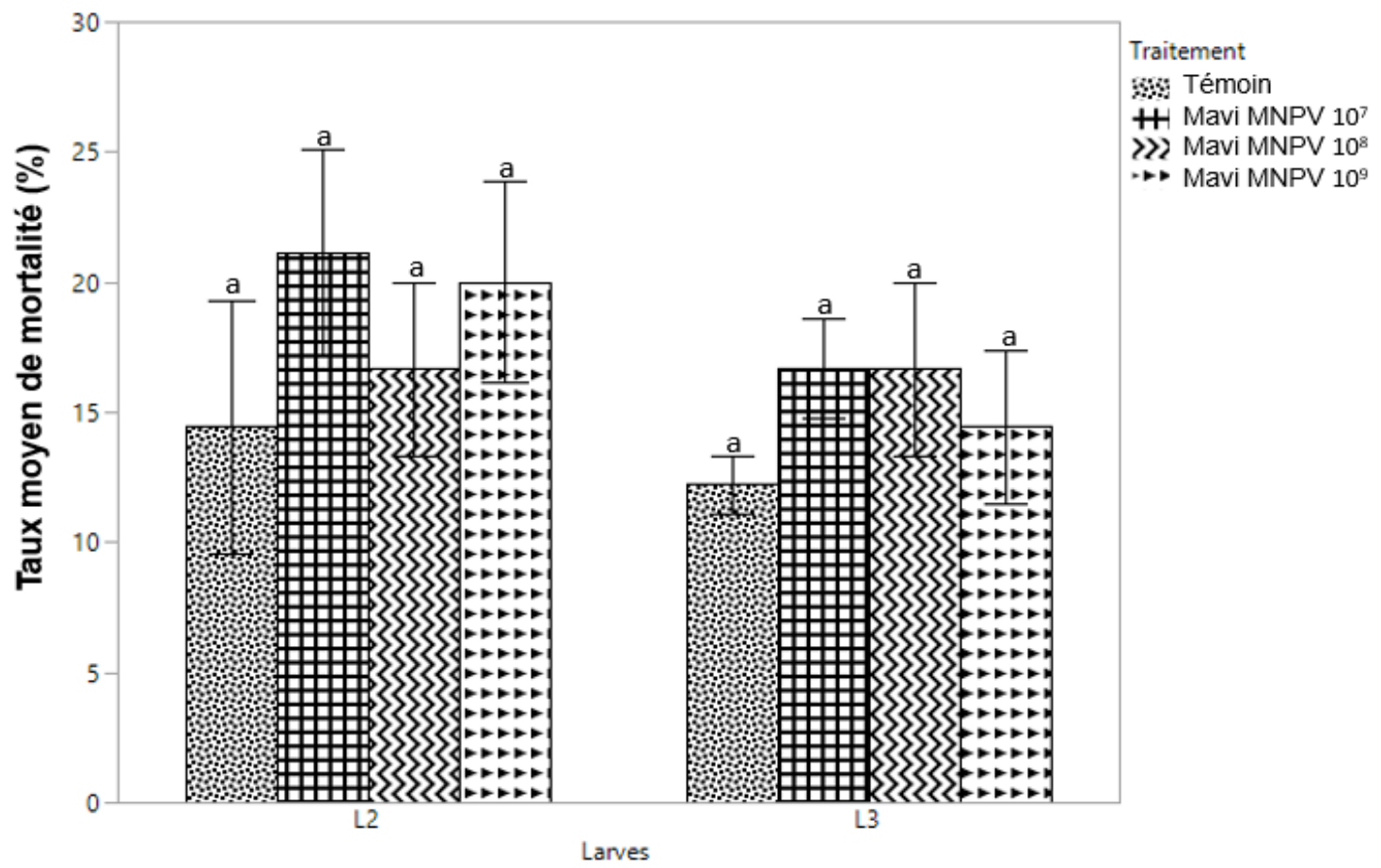

Figure 5 : Taux moyens de mortalité des larves $\mathrm{L}_{2}$ et $\mathrm{L}_{3}$ sur le substrat de mélange de Feuilles, Racines et tiges. 


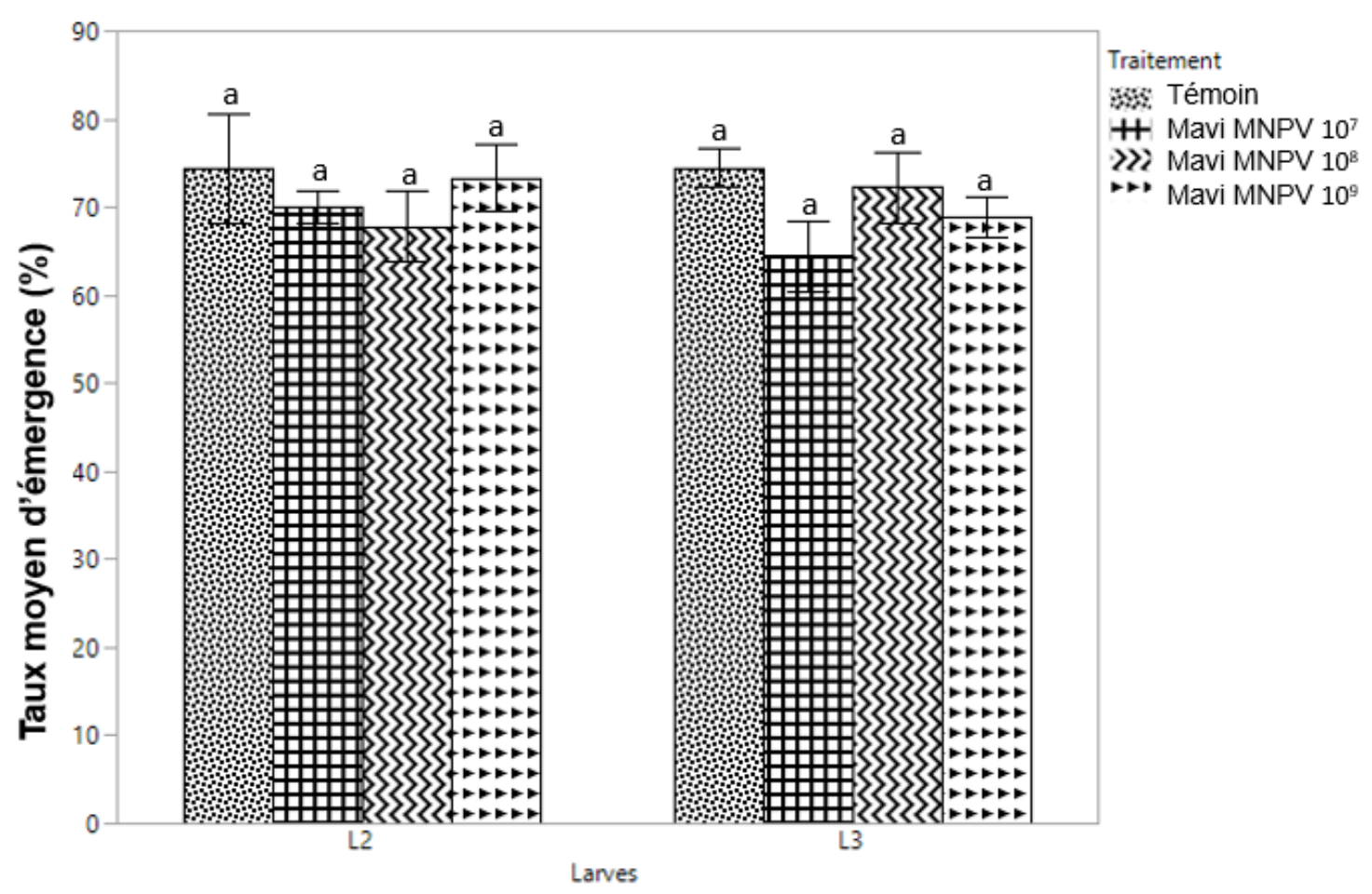

Figure 6: Taux moyen d'émergence adultes issus des larves $L_{2}$ et $L_{3}$ sur le substrat du mélange feuilles, racines et tiges.

\section{DISCUSSION}

Les données collectées dans cette étude ont permis d'évaluer l'effet résiduel de Mavi MNPV dans la nature. Les résultats de cette étude montrent que le biopesticide Mavi MNPV à différentes concentrations n'est pas persistant dans la nature. Chacune des formulations des différents substrats n'a pas affecté la mortalité d' insecte étudié. Le sable, les racines, le mélange de feuilles, de tiges et les graines prélevés après application des biopesticides au champ ont induit des taux de mortalité très faibles et similaires sur les larves de $M$. vitrata aux stades $\mathrm{L}_{2}$ et $\mathrm{L}_{3}$. Cependant, sur les larves témoins non traitées et les substrats des parcelles ayant été traitées, il y a également de mortalité. Ce qui permet de suggérer une mortalité non liée aux résidus de pesticides dont les parcelles ont reçu de traitement aux différents biopesticides. L'analyse de variance selon le test SNK au seuil de $5 \%$ a révélé que le taux d'émergence des adultes ne diffère pas significativement d'un traitement à un autre et au témoin. Quels que soient les traitements, le taux d'émergence de $M$. vitrata est très élevé ; ce qui signifie que la variation de la concentration de Mavi MNPV ne conditionne pas sa présence dans le sol, les tiges, les feuilles et les racines. L'absence de différence significative sur les faibles taux de mortalité des larves aussi bien des témoins que celles ayant reçu les substrats semble s'expliquer par leur faible temps de rémanence et absence de résidus dans les graines, feuilles et tiges. Les caractéristiques principales des biopesticides à base de baculovirus sont: la spécificité, la haute virulence, la rapidité d'action et le niveau raisonnable de persistance dans l'environnement (Dent, 1991). Cependant, les insecticides organophosphorés (pirimiphosméthyl, dichlorvos, malathion) utilisés pour le traitement des locaux de stockage vides et pour le traitement des céréales stockées présentent des résidus de pesticides dans les graines et les 
huiles brutes (Dauguet et al., 2006). De même, Contrairement à Mavi MNPV, les produits chimiques provoquent l'intoxication des utilisateurs, des consommateurs et la pollution de l'environnement (Regnault-Roger, 2002). (Ekesi, 1999) a montré que ces produits chimiques utilisés de façon accrue et inappropriée ont engendré divers effets néfastes sur l'environnement, les animaux et l'homme en laissant des résidus, dans l'organisme humain, le sol et sur les plantes traitées. Nos résultats corroborent ceux de Paynes (1982) qui stipulent que les baculovirus, constituent une solution de rechange aux méthodes chimiques de lutte contre les ravageurs des cultures. Les principaux avantages de la plupart des baculovirus résident dans le fait qu'ils sont d'origine naturelle et qu'ils sont extrêmement spécifiques à l'insecte hôte. Contrairement aux produits antiparasitaires à plus large spectre, les baculovirus n'affectent pas certains insectes, les oiseaux, les poissons, les mammifères ou les humains (Paynes, 1982). Les substrats de l'application du baculovirus dans les champs après la récolte ont tué les larves de $M$. vitrata avec la même ampleur que le témoin n'ayant pas reçu de Mavi MNPV; ce qui est en adéquation avec les différents tests exécutés par Possée et al. (1993) qui ont attesté la non-toxicité des baculovirus pour les mammifères et même les formes recombinées. Ils constituent alors des agents de lutte sécuritaires préservant la santé des vertébrés et entraînent des impacts environnementaux très négligeables.

\section{Conclusion}

L'étude menée montre que le niébé traité au champ avec ce biopesticide ne présente pas de trace du produit. Le producteur peut consommer les feuilles et les graines de niébé traitées sans se soucier de l'intoxication alimentaire. Les producteurs qui ont accès à des technologies alternatives aux pesticides chimiques de synthèse et scientifiquement prouvés, efficaces et compatibles à la santé humaine et à l'environnement. Ils ont besoin de solutions/technologies facilement applicables et à moindre coûts pour une gestion durable de M. vitrata.

\section{REMERCIEMENTS}

Les auteurs expriment leurs sincères remerciements à l'Institut International d'Agriculture Tropicale (IITA) pour son assistance scientifique et financière. Ils remercient les techniciens du Laboratoire d'entomopathologie de l'IITA-Bénin et ceux de la section niébé pour leur assistance technique et leur franche collaboration.

\section{REFERENCES}

Atachi P, Desmidts M, Durnez C. 1984. Rapport d'investigation sur les insectes parasites du niébé (Vigna unguiculata (L.) Walp) en République du Bénin (19751982). P.37.

Bass C, Denholm I, Williamson MS, Nauen R. 2015. The global status of insect resistance to neonicotinoid insecticides. Pesticide Biochemistry and Physiology, 121: $\quad$ 78-87

DOI: 10.1016/j.pestbp.2015.04.004

Ben R, Sue EH, Jenny SC, Rosemary SH. 2005: The role of plant and pathogeninduced behaviour in the persistence of a nucleopolyhedrovirus. Journal of Invertebrate Pathology, 88 : 49-57. DOI: 10.1016/j.jip.2004.09.005

Carlos JSP. 2006. Exposition humaine aux pesticides: un facteur de risque pour le suicide au Brésil ». Vertigo-La revue en Science de l'Environnement ; 7-18. DOI : https://doi.org/10.4000/vertigo.2098

Dauguet S, Lacoste F, Ticot B, Loison JP, Evrard J, Bouchtane B, Soulet B. 2006. La filière oléagineuse se mobilise autour de la problématique des résidus d'insecticides. OCL, 13(6): 373-377. DOI: https://doi.org/10.1051/ocl.2012.0476

Deguine JP, Ferron P. 2004. Protection des cultures et développement durable : bilan 
et perspectives. Courrier de l'Environnement de l'INRA, 52: 57- 65.

Dent D. 1991. Insect Pest Management. Ed. CAB International, redwood Press LTD: Wiltshire U.K., 1-12; 373-396.

El-Jasser HCA. 2011. Chemical and biologie properties of local cowpea seed protein seed protein grown in Gisan Region. International Journal of Biological, Biomolecular, Agricultural, Food and Biotechnological Engineering, 5(8): 466472.

DOI: https://doi.org/10.5281/zenodo.1078332

Ekesi S. 1999. Insecticide resistance in field populations wasp of the legume podborer, Maruca vitrata Fabricius (Lepidoptera: Pyralidae), on cowpea, Vigna unguiculata (L.) Walp. in Nigeria. International Journal of Pest Management, 45: 57-59. DOI: https://doi.org/10.1080/09670879922805 8

FAO. 2002. International Code of Conduct on the Distribution and Use of Pesticides, Revised Version adopted by the Hundred and Twenty-third Session of the FAO Council in ovember 2002, FAO, Rome, $35 \mathrm{p}$.

Gomgnimbou APK, Savadogo PW, Nianogo AJ, Millogo-Rasolodimby J. 2009. Usage des intrants chimiques dans un agrosystème tropical : diagnostic du risque de pollution environnementale dans la région cotonnière de l'est du Burkina Faso. Biotechnologie, Agronomie, Société et Environnement, 13(4) : 499-507.

Liao CT, Lin CS. 2000. Occurrence of legume podborer Maruca testulalis l'utilisation des pesticides et limiter leurs impacts environnementaux. Expertise scientifique collective, synthèse du rapport, INRA et Cemagref (France), p.64.

Narayan S, Liew Z, Bronstein JM, Ritz B. 2017. Occupational pesticide use and Parkinson's disease in the Parkinson Environment Gene (PEG) study.
Environment International, 107: 266273.

https://doi.org/10.1016/j.envint.2017.04. 010

NRI. 2012. Aperçu général de la lutte biologique intégrée dans les pays en développement, Natural Ressources Institut, Chattham, p. 20.

Ouédraogo E. 2004. L'utilisation des insecticides naturels dans la protection des cultures au Burkina Faso. Communication faite au CTR de Proceedings of the International Congress of Entomology 2008, Durban, South Africa.

p.

621. http://www.ice2008.org.za/pdf/proceedin gs.pdf.

Paynes CC. 1982. Insect viruses as control agents. Parasitology, 84: 35-77. https://doi.org/10.1017/S0031182000053 609

Phillips R, McWatters K, Chinnan M, Hung YC, Beuchat L, Sefa-Dedeh S, SakyiDawson E, Ngoddy P, Nnanyelugo D, Enwere J, Komy N, Liu K, MensaWilmot Y, Nnanna IA, Okeke C, Prinyawiwatkul W, Saalia F. 2003. Utilization of cowpea for human food. Field Crops Research, 82: 193-213. DOI: https://doi.org/10.1016/S03784290(03)00038-8

Possée RD, Hirts M, Jones JD, Bishop DHL, Cayley PJ. 1993. Fields: principaux ravageurs du niébé, Vigna unguiculata (L). Walp. (Fabales : Fabaceae). Problèmes et perspectives. Dans l'actualité scientifique : La lutte antiacridienne. Edité par Abderrahmane Essaid. John Libbey Eurotext: Paris; 269278.

Ramezani M, Oliver DP, Kookana RS, Gurjeet G, Preston C. 2008. Abiotic degradation (photodegradation and hydrolysis) of imidazolinone herbicides. J. Environ. Sci. Health. Part B, 43: 105-112. https://doi.org/10.1080/03601230701794 968 
Regnault-Roger C. 2002. De nouveau phytoinsecticides pour le troisième millénaire. In Biopesticides d'Origine Végétale, Philogène BJR, Regnault-Roger, Vincent C (eds). Lavoisier-Editions Tec \& Doc : Paris ; 19-39.

Hénault-heltier L. 2015. Health and environmental impacts of pyrethroid insecticides: What we know, what we don't know and what we should do about it. Executive summary and littérature review. Équiterre. Montréal, Canada. $68 \mathrm{p}$.

http://www.equiterre.org/publication/rev ue-de-litterature-sur-les-impacts-desinsecticides-pyrethrinoides-sur-la-santeet-len

Seri-kouassi B, Aboua L, Bekon K, Glitho A, Gérard K, N'Guessan Y. 2004. Action des huiles essentielles de deux plantes aromatique de Côte d'Ivoire sur Callosobruchus maculatus F. du niébé. Chimie, 7: 1043-1046. DOI : https://doi.org/10.1016/j.crci.2003.12.03 1

Sokame BM, Tounou AK, Datinon B, Dannon EA, Agboton C, Srinivasan R, Pittendrigh BR, Tamò M. 2015. Combined activity of Maruca vitrata multinucleopolyhedrovirus, MaviMNPV, and oil from neem, Azadirachta indica Juss and Jatropha curcas L., for the control of cowpea pests. Crop Protection, 72: 150-
157.

DOI:

https://doi.org/10.1016/j.cropro.2015.03. 016.

Tamò M, Srinivasan R, Dannon E, Agboton C, Datinon B, Dabire C, Baoua I, Ba M, Haruna B, Pittendrigh BR. 2012. Biological control: a major component for the long-term cowpea pest management strategy. In Innovative Research along the Cowpea Value Chain, Boukar O, Coulibaly O, Fatokun C, Lopez K, Tam_o M (Eds). Proceedings of the 5th World Cowpea Research Conference, 26 September -1 October 2010 Saly, Senegal, p.249-259.

Toffa Mehinto J, Atachi P, Elégbédé M, Douro Kpindou OK, Tamò M. 2014. Efficacité comparée des insecticides biologiques et chimiques dans la gestion des insectes ravageurs du niébé (Vigna unguiculata) au Bénin. Journal of Applied Biosciences, 84: 7674-7681. DOI: https://doi.org/10.4314/jab.v84i1.1

Van Lenteren JC, Bale J, Bigler F, Hokkanen HMT, Loomans AJM. 2006. Assessingrisks of releasing exotic biological control agents of arthropod pests. Annual Review of Entomology, 51: 609-634. DOI: 10.1146/annurev.ento.51.110104.151129 